Voix et Images

\title{
Sur le trajet poétique de Fernand Ouellette
}

\section{Pierre-Justin Déry}

Volume 5, numéro 3, printemps 1980

\section{Fernand Ouellette}

URI : https://id.erudit.org/iderudit/200228ar

DOI : https://doi.org/10.7202/200228ar

Aller au sommaire du numéro

\section{Éditeur(s)}

Les Presses de l'Université du Québec

\section{ISSN}

0318-9201 (imprimé)

1705-933X (numérique)

Découvrir la revue

\section{Citer cet article}

Déry, P.-J. (1980). Sur le trajet poétique de Fernand Ouellette. Voix et Images, 5(3), 497-513. https://doi.org/10.7202/200228ar d'utilisation que vous pouvez consulter en ligne.

https://apropos.erudit.org/fr/usagers/politique-dutilisation/ 


\section{Sur le trajet poétique de Fernand Ouellette}

\section{Ces anges de sang (1955)}

Un texte bref, découpé souvent en quelques strophes concentrées, parcourt fiévreusement les premières pages de Fernand Ouellette. La narration y trouve déjà le langage abstrait qui caractérisera presque toute son couvre poétique à venir. Dès l'abord, l'humanité se fusionne au mûrissement végétal en un étincellement de fruit noir. Incendiaire, le vent onirique connaît un instant la portée divine de la lumière. Promesses de l'illumination et de la parole que le poète confie à un corps en croissance, en projet, en densité bourgeonnante. Le verbe de l'oracle s'ouvre vers les ramifications de sa science, de sa prescience et de sa conscience: «Dans l'homme je sais un vent mûr" (Poésie, p. 13). Toutefois, l'inaccessible métaphysique menace d'interrompre le dynamisme spirituel en absorbant l'élan venu des profondeurs. Seul principe fondamental du visible, le soleil parvient encore à valoriser l'œil et la montée malgré la désespérante absence. Car s'instaure déjà la thématique de l'âme déchirée entre l'immanence et l'utopie. Générateur du rêve et de l'idéal, l'esprit se nourrit du principe igné, et ce jusqu'à l'excès, jusqu'à la destruction du principe matriciel de la mer, jusqu'à la pétrification du navire exploréen.

La naissance de la musique cherche son lieu dans le creuset de la parole, à travers la crémation de la vie et de la mort conjointes. Si le diurne peut ravager la paix intime en dénudant la conscience, le nocturne favorise au contraire les motifs de lîle, du lieu enclos et de la vision séraphique. Car les yeux qui guettent, vigilants les signes de l'univers, sont blessés par les funestes présages de la cendre. Le regard fécondant conserve le pouvoir de rénover le milieu brûlé par l'intensité meurtrière du soleil, pourvu que s'accomplisse la végétale poussée vers l'idéal. Mais lorsque la stérilité crève la pupille et abat l'oiseau, ce n'est pas la cécité qui touche le poète, mais au contraire la voyance: la fulgurance intime donne accès au secret, au passé et à la réminiscence. Et même l'aveugle confronté à l'enfer des ombres découvre la rédemption du temps griffu par la méditation du mouvement perpétuel, du temps cyclique. Dans cette poésie, en effet, rien n'est jamais assuré définitivement et le jeu permettant des antithèses ménage le maintien d'une tension indispensable tant à la subversion de l'onirisme qu'à sa communion avec le réel. 
S'il arrive maintes fois que le poids de ce réel brise la vision partielle du rêve, ou que le mordicant s'immisce au centre du lieu paradisiaque de la nidification. Fernand Ouellette scelle soigneusement le pouvoir incantatoire de sa création dans les veines, l'âme et l'utérus du cosmos. Au-delà de la terreur, du temps, de la diversité qualitative, des inégalités, de la mort et de l'oubli, se perpétue l'intervention divinatoire de l'homme. Son invocation à l'ange exige la restitution d'un corps délivrè de sa vaseuse religiosité, réclame une ardeur et un dynamisme habitables à la fois par la spiritualité et la matérialité: “Quel ange me rendra / le haut sentier d'un geste plein / l'ardent pays d'un corps en marche?" (Poésie, p. 21). Certes le présent ne manifeste guère cette chair glorieuse à venir. Tout au plus permet-il à l'être oppressé par le silence et le sang, engagé dans le tunnel du froid, engouffré vers les multiples morts des orgies et des soifs, de croire encore. Croire en la rédemption du pays solaire, à l'exotisme des palmes, au rivage de la iraternité. Quant au passé, il tue le futur, révélant le gel qui entrave la joie de la parole. Le bourgeon, l'aile et le désir motivent la montée de l'ange, mais l'humain ne parvient pas à suivre une telle spirale. Le soleil avorte à l'orée de l'œil.

Et voici que le messie venu à travers la souffrance offrir le salut, troquer le sang contre le sable, substituer l'avenir au passé, voici que lui-même se pétrifie parmi les fossiles. L'ange de feu est sacrifié à la cendre, le nocturne enraye la conquête du diurne. La dessiccation attaque le vent, l'aube, le pivot entier du monde, condamnant à la solitude l'homme qui s'évacue de la passion, de l'éclair de la chair, pour accéder à l'au-delà. Maintenu sans cesse dans le projet, dans le trajet, l'être s'affranchit pour un temps des tourments de l'immanence. La neige dans le nid natal se change en plume-feu-regard. La lumière se joint à la chaleur dans une présence odorante de la femme qui attise l'aile, la transcendance et la parole. Mais la plainte et les sanglots témoignent bientôt de la déchirure qu'impose l'abandon de la mer pour l'exil solaire et le dôme de pure sonorité. L'instinct de l'animal comme la vie fouisseuse du terrier altèrent la figure sacrée du prophète, dramatiquement coupé de son élan vers l'éternité. Le feu diurne se résorbe dans la terreur de l'abîme et l'universelle souffrance. L'ultime refuge de l'humain réside dans le souvenir de la parole divine et l'espoir en la résurrection.

Fernand Ouellette poursuit la lucidité jusqu'à la révélation de la stérile marche de l'humanité. S'enfonçant vers le nocturne et la solitude, l'esprit se perd en un lieu clos et s'épuise dans l'assèchement: "Lucidement / nous épuisons dans nos pas la montée des nuits, / nos pas brisés sur les traces de l'homme." (Poésie, p. 34). Seul l'effroi devant la mort parvient à ranimer l'immémoriale blessure et à provoquer le retournement total de cette quête. Ainsi, à la toute dernière page du recueil, ressurgit le chant paradisiaque et la promesse d'une vie ouranienne. L'articulation du «mais" rend possible une rupture qui projette l'événement dans le futur, dans l'utopie. Revalorisés par l'altitude et le ciel, protégés par l'enceinte des mains, l'œil et l'élan se trouvent finalement accordés dans le jaillissement vers l'absolu. Car c'est l'errance qui définit l'avènement de l'unité, enfin réalisée dans la mouvance du devenir, hors du statisme du être. 


\section{Séquences de l'aile (1958)}

Incursion dans la modernité et le concret, la démarche de ce second livre reste à certains égards décidément distincte de celle du premier, ainsi que de tous ceux qui suivront. Cette différence est d'ailleurs sensible à plusieurs niveaux: thématique, syntaxique, stylistique, idéologique, etc... Sa structure elle-méme a été considérablement modifiée dans la version que l'auteur en donne dans Poésio (livre qui regroupe tous les recueils publiés par Ouellette de 1955 à 1967, souvent remaniés, ainsi que des inédits de 1968 à 1971 , et auquel réfèrent les citations de la plus grande partie de la présente étude). L'insurrection de l'âme y force le recours à une aile paradoxalement nocturne et tellurique, à un ange de terre qui porterait la destinée de l'humanité. Tel se dessine du moins le projet à travers les deux épigraphes mises en tête du recueil (p. 41). La solitude de l'être confronté au chaos et au dualisme cosmique va soudain se métamorphoser en une solidarité avec le milieu réunifié. Frappé par la lumière, le vivant est transmué, rapatrié dans la rumeur du monde. L'animalité décline en lui à mesure que s'accroît la spiritualité qui déleste l'homme de ses illusions.

Soumises à la raison, la sensualité et la passion auront désormais droit de cité dans un milieu où le corps, la musique et le végétal se voient allégés par l'auréole et l'ascétisme. L'envol dans la transparence dynamise le feu galactique et sexualisé, façonnant une réalité toute nouvelle parce que le regard qu'on lui porte est neuf. Alors l'oiseau peut imposer l'anti-gravitation, la chute vers le haut, et ainsi délivrer l'arbre de sa condition d'enraciné, le sauver: «Tout le délire d'un regard, la gravitation d'un oiseau déliant les arbres" (p. 45). Cette gravitation inversée incite Fernand Ouellette à célébrer l'épopée de l'altitude, l'ivresse du vertige de l'homme ailé. Par un tel renversement, la terre elle-même est entièrement bouleversée, régénérée. Échappant à l'angélisme, l'oiseau igné sait réinsérer la vie lumineuse dans le cadre chthonien. II marque une nouvelle naissance, bien sûr, mais aussi pénètre et transcende rituellement la mort. L'allégement projette la transfiguration et recrée l'élan et la progression universels. Le redressement de l'esprit entraînant celui du corps, l'impulsion spatiale génère le désir sexuel qui, souverainement, rétablit l'équilibre entre l'horizontalité et la verticalité.

Victorieux de la mort, l'ange et l'air s'associent au vent pour remonter l'axe du monde jusqu'au refuge altier. Le ciel atterrit dans le sensuel pendant que la terre s'envole dans le sanguin et la musique. Voici donc retrouvée l'harmonie dans la conjonction ciel-terre / vertical-horizontal. Par la lueur et la parole s'accomplit l'euphémisation du passionnel et du catamorphe, l'exorcisme du sang. Même la mort se désagrège sous la provocation du feu, de l'ange et de l'aile: l'essence recouvre enfin la paix phénoménologique. Cependant, l'érotisme va reconstituer la douleur et la déchirure au sein de cette unité primordiale, reconstruisant le dualisme ange-sexualité. La main caressante et marine meurt sous le choc ornithomorphe et porteur de verticalité. Le vertige, l'œil, la transparence, l'aile, contrecarrent les doigts, la 
peau, les mains, les cheveux, les bras, les lèvres, tout le vivant aspiré à nouveau par l'éther. Dès ce moment, l'Éros réintègre le principe de la dualité, la transparence de la psychée se heurtant à l'opacité organique. S'amorce l'exploration du précipice de la séduction, de l'obscénité et du désir, par le rejet du rouge sexué et nocturne ainsi que du faux soleil de la pilosité. La castration par la chasteté atteint les nébuleuses.

Quand le sexe ombreux signifie chaleur, encerclement, germination et calme habitat, c'est que l'extase féminine demeure censurée par la lumière. La mort disparaît parce que la clarté angélique enraye la ténèbre du mâle. Axée vers le polaire vivifié, la matrice se purifie au contact du froid cosmique et de la blancheur. Evidemment, semblable métaphysique du sexe ne peut qu'engendrer l'échec, "le mal de vivre"(p. 52). Cette fois encore, l'unique rédemption réside dans le flambeau et le son qui transgressent l'abîme. Par l'ceil, le vol rehausse le poète au-dessus de la pétrification. Le dynamisme solaire, ailé et spirituel, s'oppose à l'immobilisme charnel et osseux. Cette possibilité de salut par l'envol élabore d'ailleurs la transition vers Radiographies, qui formait originellement partie intégrante de Séquences de l'aile, et que Fernand Ouellette a choisi de démarquer comme recueil distinct dans Poésie. Dans le premier texte de Radiographies, l'homme remonte tout le tangible jusqu'au vaste espace. Le sang émotif se rationalise en cerveau; le coeur lui-même bat à haute altitude, hors du temps et de l'espace réunifiés en la nouvelle cosmogonie. Le dégel ranime le passé et le futur en un présent privilégié, accélérant la distorsion de l'environnement, l'expansion du moi et l'accroissement continu du monde.

Mais tous les poèmes qui suivent opèrent un saisissant revirement de la volonté verticale qui, plutôt qu'une ascension et une concentration, propose un enfoncement et une dispersion de l'être. Exacerbé, le désir s'embourbe et se fige dans l'impuissance et la destruction. Du cerveau incendié surgissent des sons et des parfums hybrides, excessifs, menaçants parce que générés par la nuit. Le sexe devient spectacle. Entraînant la souffrance et la mort, il court-circuite le devenir universel. Vision désespérée, désabusée, d'une civilisation dont la désolation emprunte les attributs du bordel et du cirque, où l'humanité mécanisée a perdu son âme. Dans ce parti pris de modernisme urbain, l'atome, la Bourse, le cérébral remplacent l'affectif. La mort du troupeau mi-animal et mi-humain se poursuit dans la dessiccation et le silence. Le cœur et le verbe se synchronisent avec la glace, la musique percutante d'Edgard Varèse ponctue la révélation apocalyptique d'une ultraurbanisation du paysage physique et psychique. La paralysie et l'impuissance, la culpabilité et la fólie, la mutité et la sécheresse, tout concourt à l'ossification ainsi qu'à la fin d'un monde ailé. Puisque l'enfer moderne occulte toute lumière et tout mouvement, le fractionnement intime oblige à la condensation vers l'essentiel noyau de l'être, puis à son explosion pouvant seule offrir une nouvelle promesse d'ascension. 


\section{Le soleil sous la mort (1965)}

Dans son troisième recueil, Fernand Ouellette marque un retour à l'esthétique de la rigueur et de l'abstraction du premier livre, mais sans se départir d'un certain engagement socio-politique découvert au cours du second. Le poète approfondit même, tout au contraire, l'intensité de son propos ainsi que la concentration de son expression. L'individualité, la collectivité et l'historicité se coordonnent afin de remythiser la progression de l'humanité vers la jonction du besoin passionné et de la conscience lucide. Voici fusionnés l'ombreuse densité du présent et le lumineux infini du passé et de l'avenir. L'expérience politico-historique du peuple déploie l'expérience érotico-spirituelle de l'intimité, comme en fait foi la phrase de Gaston Miron mise en épigraphe à la première partie, En lumière. Le pays s'y révèle à la fois amer et matriciel, territoire de l'amante et de la mère. La plupart des textes de cette partie sont d'ailleurs rédigés au passé (imparfait, passé simple, passé composé), ce qui semble indiquer que le rêve d'une humanité baignant dans l'édénique illumination relève d'une époque bien révolue, ne rendant que plus sensible l'amertume du présent.

Le peuple erre vers la mort, sous le joug de la souffrance et de la répression. Attaqué par la corrosion du désir, de l'œil, de l'amour et de la chair, il s'enfonce dans la folie. La race saigne et le " nous " collectif qui jaillit de cette sève juxtapose les multiples solitudes en un cheminement authentique vers la solidarité. Enfouie sous la terre, la flamme réclame l'enfance, de même que le soleil enfermé sous l'eau appelle le múrissement du jour et l'accomplissement du printemps. Cette libération d'une véritable parole se célèbre donc dans le cri : "Ainsi nous parlions avec une voix en naissance qui hurle» (p. 86). Le feu au centre de la chair provoque un éveil dynamique du peuple dans le blanc, cette marche idéale vers la vie et l'amour. Mais semblable temps béatifique se trouve désormais banni d'un univers où l'humain se voit pillé par les mots et la raison des fourbes. Sous la domination d'un maître dont la langue lui est étrangère, le peuple reste impuissant à assumer la vie. Seuls le rêve et le cri laissent présager que sa soumission ne demeure pas irréversible, peut-être.

L'homme d'ici a connu un passé de calme agonie, une marche nocturne où il se refusait pourtant au sommeil, guettant la blessure illuminante, la torture ignée, attendant que son rêve de liberté se réalise. Aujourd'hui, cette Amérique se dresse dans sa nudité et sa blancheur natales, quittant le souvenir pour la promesse matricielle de l'œil et du végétal. Car c'est l'arbre qui, comme l'homme, supporta la brûlure, la mutité, la ténèbre et le ravage de tout. Et soudain, par l'intervention de la parole revendicatrice, l'humain et le végétal se trouvent réconfortés d'une même chaleur. La verticalisation du principe même de l'horizontalité, le fleuve, démontre ce pouvoir d'évolution et d'allègement caractéristique de l'arbre axial chez $F$. Ouellette. La régénaration de l'individualité entière s'accomplit par l'accession à la lumière et au sang. L'œil s'ouvrant à l'espace, le verbe redevenant sacré et fécondant, la dynamique de l'organisme épouse celle de l'arbre. Malgré le souvenir de son 
accablement, l'être collectif se sait promis à un avenir igné et aqueux à la fois, pacifiè par l'amour et le végétal. Dans une tension dialectique salvatrice, la chair croît hors de la mort, la glaise, l'obscur, vers le jour, l'air, le blanc. Le silence se permute jusqu'au cri, l'ange sépare l'essence et la matière, et le sang, l'amour et le corps sont sublimés dans l'assomption.

Hantée par le spectre d'un désastre nucléaire, l'historicité de L'absence du soleil, deuxième partie du recueil, s'inscrit profondément dans la violence cosmique qui imprègne le temps présent. La mort frappe de plein fouet le héros solaire, l'amour et la blancheur étouffent sous l'angoisse des cendres. Asséchant le passé méme, la nuit et le silence perforent tout le réel. Certes, Fernand Ouellette ne se ferme pas à l'espérance: l'enfant, le Christ, le divin et l'amour pourront triompher. Mais, pour l'instant, le printemps semble impuissant à percer le gel, la parole ne se dresse pas au-dessus du noir et l'ange s'animalisant, se matérialisant, ne pénètre pas le jour. Comme l'esprit, le macrocosme indivis et le microcosme atomique signalent la complète abdication de la vie. Exsangue, pétrifié, enténébré, le cœur en quête de soleil et d'onirisme ne trouve que le néant. Les forces apolliniennes du cerveau, du regard et de l'esprit se heurtent aux mêmes limites, car l'air est fermé, l'espace gelé, l'infini torréfié. Le son et la lumière, le végétal et le solaire, deviennent impuissants à dire la sacralité. La signature divine est scellée dans une parole incommunicable: «Le lilas est aveugle, le soleil illisible» (p. 106).

Cécité, ossification, putréfaction, cendre, silence, autant d'indices du suicide cosmique. Alourdie, la terre se fatigue; de même, privé de l'aile et du ciel qui conféraient l'élan aérien à son horizontalité, le fleuve se dé-verticalise; enfin, expulsé du désir, le phare se décompose. Ainsi, les principaux éléments forment une monstrueuse mégabombe de neige où s'amenuise la nécessaire clarté. Le temps saigne dans le noir: Dieu, l'ange, le regard, la bouche, l'enfant, le sein, la femme, la bête, l'homme, l'amour, la parole, le végétal, l'air, tout l'idéal spirituel et charnel est déserté et enténébré. L'esprit ici laisse s'insinuer, le mal, la mort et la désincarnation. L'humanité abandonnée par un dieu en pourriture, soumise à la guerre, se rassemble dans les hauts lieux des souffrances collectives pour exorciser le malheur. Au cours de la régénération cyclique, la vie appelle la vie. Le cataclysme guerrier suscite certes la bestialité du peuple coupé de l'essentielle tendresse. La terre fomente l'ossification, le noir et le silence, lorsque le cri de la révolution prononce la mort du brasier dans ala nuit de l'homme. Mais la puissance incantatoire du verbe poétique réveille l'espoir en la fraternité et le bonheur. Une race nouvelle revivifie la verticalité, redonnant la parole, la fécondité et l'ouverture vers le futur. Dans le jour de l'homme, le présage inaugure le possible destin de l'enfant.

Quant à la troisième partie, L'amour solaire, elle exprime la foi que l'amour puisse survivre aux énergies de la mort. En un douleureux acte de naissance, la chair se valorise en corps musical pour accompagner le printemps et l'aube dans une tentative de réunification matière-esprit, glacefeu, obscurité-vie. Et F. Ouellette retrouve ici cette tension constante, cette 
polarisation indispensable au rayonnement de sa poétique dans la montée et l'élan: "La vie monte aux vertèbres $/(\ldots)$ vers le pôle son visage s'élance» (p. 121). Au-delà de la destruction, donc, le désir enchâsse l'humain dans la permanence, lui permettant de surmonter l'angoisse du déterminisme. Car la lumière préserve l'idéal onirique du noir faisceau du réel; la renaissance de l'astre assure le dynamisme cosmique et végétal. Victoire ignée de la vie et du sacré, certes, d'où célébration des noces mythiques Soleil-femme, mais aussi, par la médiation de l'arbre axial, reconquête de la transparence, de l'aile et de l'amour soudain purifié. Dans la blancheur diurne, l'œil envahit enfin le monde, revitalise les morts, offre la terre à l'entière cosmogonie. Du même souffle, la femme de matière, lourde, sombre, parvient à la verticalité et à l'allégement par l'iris en croissance qui garantit sa luminosité. Cependant, son émergence dans le blanc demeure momentanée, éphémère, le temps de solariser l'homme. Car dès que le désir n'est plus latent mais s'actualise plutôt, se réalise, il perd un peu de la noblesse que lui conférait son immatérialité.

Et déjà l'union sexuelle appesantit à nouveau l'être double qui sacrifie son élan spirituel à l'épaisseur de l'Éros, préfigurant toute la tension thématique de Dans le sombre. C'est d'ailleurs à une exploration plus minutieuse de la synthèse de cet érotisme et de cette spiritualité qu'invite déjà la dernière partie du recueil, Naissance de la paix. Pendant que le blanc et l'aile repoussent la nuit, l'esprit règne dans la pureté, la virginité et l'absence de violence. L'amour se hausse beaucoup moins facilement au-dessus de toute gelure, ne réussissant à s'intégrer à la terre et à la chair qu'au prix de la souffrance. Cette douleur de l'enfantement convoque la passion de Marie, le Verbe christique, hors du plaisir érotique. Car la sexualité, vécue sans élan vers l'envahissement du prégnant désir qui la transcenderait, ne peut apporter que la pétrification du propos. La matière doit donc se transfigurer par la purification du feu, de la montée et de l'air, même au risque de basculer dans la mort. Le grand mutisme de la certitude du temps et du fini va en effet préparer la parole sacrée de l'Étre, du Christ et de la Paix.

\section{Dans le sombre (1967)}

La mort dans la lourdeur, la paralysie et le silence, cède la place, dans le quatrième livre de Fernand Ouellette, à une mort par le plaisir sadique, la délirante volupté, l'angoisse toujours désirante. Quant à la réalité historique du monde, elle se voit détournée, transcendée, et en un sens assumée, par l'expérimentation dialectique d'un au-delà de la psycho-physiologie. Cet Éros, comme conscience abstraite du corps, ou jugement calme porté sur la démence du sensuel, focalise donc plus que jamais la tension poétique en quête d'infini dépassement vers l'absolu et le métaphysique. Une mystique des sens s'élabore dans la violence, car le plaisir sexuel ne suffit pas à animer l'être, à le délivrer de son horizontalité. Au contraire, l'aile noire et la glaise, le ciel alourdi sur le sexe forment autant d'indices de la morbidité des sens. Les notions de rationalité, d'effroi, de destruction, 
de piège, de chute, de cri, etc., ravivent la conception de la faute adamique. Enfin la forme sexuée se donne physiquement à la chaleur et à la lumière, mais l'être est divisé et l'esprit exclu de la fête. Tour à tour igné, végétalisé, irradié, le corps se coupe de l'aile et plonge amèrement dans la nuit.

Dès lors, le végétal et le minéral contaminés par le désir regagnent le mutisme des signes scellés par la profanation du toucher. Propageant le sombre, la femme génère la mort, la foudre et le gel. Elle provoque la fuite de l'oiseau, l'extinction de l'âme, la perte du regard. A d'autres moments, dans le délire des caresses, le regard atteint au contraire une acuité inusitée, mais toujours aux dépens de l'intégrité physique, toujours au prix de la voration et de la douleur. Ainsi l'homme souffrant sollicite-t-il les forces de son sadisme et de son agressive mordication, mais toutes les pulsions incendiaires s'en voient immédiatement désamorcées par le gel. Fasciné par la lumière et la transparence "d'un cygne / d'outre-chair" (p. 154), cette aile de la lucidité, le poète se voit malgré lui alourdi par le sensoriel. Déchiré, rejeté hors du blanc vers le nocturne passionnel, il sombre dans l'affolement. Le mâle se désagrège par défaillances successives en l'entaille qui le provoque et le choisifie. II se brûle à cette courtisane qui l'assombrit sans jamais l'accomplir. Le voici acéré, exilé de la musique et de l'espace libre, tourmenté par une irrépressible idôlatrie sado-masochiste, frustré, dépossédé, mais encore et sans cesse revenant. Inextinguible, mort et pourtant immortel, il entre illuminé « au grand tombeau de ciel» (p. 156) qui le resacralise en le sacrifiant pour le recréer phénix.

Dans la deuxième partie du recueil, l'homme retire la femme de sa tourbe comme Dieu a tiré Éve de la côte d'Adam, mais aussi comme un vêtement sorti d'un coffre. Femme-objet en même temps que signe sacré du blason intime, elle prolonge la dialectique du désir. Profanateur, Fernand Ouellette viole et brise, mais adorateur, il se violente également sans pitié. L'amante devient cette carte perforée, objet informatisé du réseau électronique que l'appranti-sorcier cherche à explorer et contrôler. Par la machine à plaisir, l'amoureux descend aux enfers de la noire jouissance, dans l'abîme des obsessions et des orgasmes, où tout le corps se démembre dans la folie ignée. Le « $O »$, matrice femelle, cercle de la femme-habitat, perdure par-delà la dissolution du "l», matrice mâle, verticale de l'homme-axe du monde.

Parce que le sexe féminin est cette blessure féconde qui éveille sadisme rituel, il menace de mort les pulsions thériomorphes qui veulent y guérir les signes du divin. La chair suscite la souffrance, la noyade, la brûlure, le froid, le fléau, le mal, l'angoisse, la panique et la démence, car la crudité excessive de l'érotisme ne peut qu'exacerber la tension spirituelle sans pour autant l'assouvir. Si le cœur lumineux, en effet, semble toucher au solaire espace de la conscience, par contre l'acte sexuel obscurcit le regard et s'objecte même à l'intégrité du sublime orienté vers l'acquisition de la connaissance.

Bien sûr l'ardeur est le vampire de l'âme dans ces moments où la vulve se fait enfer, désert, sang et nuit. Décroissent soudain l'influence de l'oiseau, 
le pouvoir de l'œil, la dynamique de la soif. Expulsé du bleu idéal, l'esprit a laissé place à l'instinct, et même quand l'attrait sensuel dissipe l'incertain, il ne redonne pas l'aile salvatrice. Anneau de noir, la femme reste un centre conflictuel, un mur de matière, et l'homme cherche vainement à l'alléger, à la «tirer du temps» (p. 170). II se condamne ainsi lui-même à la déchirure, à la mort, à l'incessante oscillation de la crainte au désir. Tournant le dos au soleil, le mâle opte enfin pour l'ombreuse femelle. Il dépouille alors l'amante de son auréole de sacralité pour la sacrifier, la muter en "fille" sombre, putain, et l'offrir au gouffre de la pathologie. Pareil choix, sans doute, ne connaît d'issue que dans le "délire désastreux" (p. 172). La femme immole à son tour l'amant, le torture, l'abêtit, le désunit de lui-même et, quoique le forçant au silence et au sang, le laisse toujours brûlant de désir. Cette prostituée close, givrée, monnayable, entretient avec celui qui la sollicite des relations venimeuses. L'indifférence, l'ennui, l'habitude, la mécanique, entraînent les amants vers la momification et la mort. Piégé, l'ange vertical et igné de la pensée vient glisser, pantelant, dans le lit glacé du fleuve sexualisé. L'agressivité animale perce la tendresse humaine quand le délire souille l'angélisme, le désamorce et l'étouffe. La virilité creuse la brèche, dévaste la courbe, dissèque le corps; la féminité réussit mieux à recouvrer la fulgurance, l'air, la lumière, mais toujours dans l'ambiguïté.

Semblable tension entre les forces paradoxales de la beauté se retrouve dans la troisième partie du livre, mais comme intervertie cette fois. L'homme nocturne et horizontal communie à la femme verticale et lumineuse qui lui transmet sa volonté de remontée, d'âme et de transparence. L'abîme se voit renversé, donc essentiellement ascendant. La douleur s'équilibre dans l'éruption, la mort charnelle côtoie la mouvance du blanc et le feu épouse l'ombre. Car dans l'unité et l'innombrable, la mort réalise la vraie vie dans une nuit illuminante où la parole touche au divin par le sexe. Le bleu idéal retrouvé effile l'axe du corps, redressant l'être et rendant possible la pénétration de la matière par l'œil. A la conquête du jour et de la gloire dans la sexualité incandescente, la volupté marque une naissance absolue oủ se concilient les extrêmes et où l'aplanissement même se fait serein. Toutefois, cet asservissement à l'immanence amorce le retour à la bestialité destructrice, mais dans une souveraine ouverture du mal sur la lumière. Le ventre s'élève vers la vision et $F$. Ouellette réalise la voyance et le salut d'outre-tombe par la volonté de non-pourrissement et de fécondation.

Les forces contraires s'annulent donc dans la régénération d'un réel absolu, par la méditation d'un infra-univers. Le feu confond au gel, la mort à la semence, la douceur à la douleur car l'amour unifie et transfigure les sensations. D'une part chaleureuse, passionnée et végétale, d'autre part immatérielle, divine et cérébrale, la femme devient le lieu même de la tension. Elle "calme et amplifie le mal " d'un seul geste, et permute le noir et le blanc (p. 187). Vierge bien ornithomorphe, elle dynamise l'entière verticalité. L'élan réapparaît enfin pour alléger le couple édénique et lui conférer la sacralité du principe pneumatique. Assumée et sereine, la copulation atteste l'indivisibilité cosmique par la clarté et le son. Toutes limites franchies, le regard, le 
silence, la montée peuvent se fusionner au pubis, à l'orage et à l'horizontal. Le corps accède à l'extase spirituelle. Le long de l'axe vertical, l'ange descend jusqu'à la sueur, dans la plénitude et la mutation du couple transfiguré dans la blancheur d'une mort supérieure. L'âme s'épanouit sous la chair, le soleil et l'enfer se rassemblent en l'illumination extatique, le coït se vit hors de cette dualité résolue par la réinsertion embrasée de l'aile: " tout oiseau, tout désir " (p. 191).

Le combat sexuel semble donc gagné. Mais le repos qui couronne cette victoire met bientôt l'être aérien en danger d'étouffement en lieu clos. Et la quatrième partie du recueil institue alors une nouvelle brisure au creux du vivant. L'aplomb végétal y adoucit le mal et la souffrance, certes, mais dans le secret, le blanc et l'immobilité. Le quotidien propageant la mort et la descente femelle, seul l'organe vif du mâle, par son principe pyromane, pourrait réinstaurer la volonté héroïque et diurne. Toutefois la soumission nocturne et intime de la femme présage l'avènement monstrueux du mutisme et de la stagnation. Déjá la brûlure s'oppose au noir, déjà l'anatomie rebute au sacré. Et le temps répand l'ennui sur l'amour par perte de chaleur, la femme ne pouvant plus assumer sa mutation ardente, sa sublimation. Reconquise par le sang passionnel, délaissant l'auréole de l'ange, elle se coupe de l'air et de l'aile. C'est pourquoi se flétrit la substance dans le froid, l'aphasie et la minéralisation. Après une dernière effusion sensuelle qui redresse l'onirisme, vite les soleils déclinent. Une démarche semblable à celle des deux premières parties du livre va maintenant se rétablir et perdurer jusqu'à la fin.

Incandescente, la femme érotique génère l'impunité du couple en favorisant la chaleur et l'illumination. Elle consacre la renaissance du principe aérien du bleu et du principe igné de l'âme dans l'éclat de l'oeil. Transcendée par l'union, la vulve redonne la totalité à l'organisme bien végétalisé du mâle qui s'élève au-dessus de la mort. L'homme se métamorphose alors en arbre solaire, tout élan et mouvement. S'attardant à la verticalité du corps féminin, il trouve son énergie dans une matérialité distendue vers l'indéterminé, ce qui transmue l'os, le gel, le sec. II forme la densité, la concentration et, par l'entaille sadique, y redécouvre l'originel. L'ange s'inscrit donc au cœur du tangible pour spiritualiser la pierre et guérir "le mal du paysage" (p. 206) par la blancheur du sang, cette équation passion / raison qui ouvre le temps d'ici à la fascination d'un illo tempore retrouvé. Quand la femme refuse la droiture, l'axe, le póle, l'homme s'atrophie en l'acte lubrique. L'aspiration et l'inspiration manquent au couple qui n'assemble pas sa chair et son esprit dans l'amour. Seule une telle volonté de divinisation par le sexe justifie l'espoir en la solarisation de l'être après la mort. La fusion des amants rend en effet possible l'accès à l'immortalité par l'infini désir, cette force de communion avec le cosmique, la musique et la lumière. Égaré dans le lieu de la passion, le mâle raisonnant jaillit bien auroral vers la ténèbre femelle, comme une bête enfermée soudain relâchée dans l'instinct.

Alors l'aile impose nettement à l'homme le supplice de l'écorchement et du mutisme. Puis la femme se substitue à l'ange pour approfondir l'entaille. 
Initiatrice, elle le libère par l'amour, l'illumine, réussit à le sauver paradoxalement à la fois de l'immobilisme de la blancheur comme de l'inhumaine perfection. L'érotisme lui recrée un corps de parole que le temps, l'usure et l'errance condamnent cette fois à une autre mort, dans la beauté de l'âme associée à la chair. Ne vivant que dans le jet, le bond, l'éclair, le poète se voit ainsi déchiré sans cesse entre les deux pôles de sa personnalité, mi-diurne et mi-nocturne. Plongé dans la luxure, il décourage les essors du végétal, de la musique et de l'oiseau. La bestialité, l'instinct, la tentation, annihilent tout dans la dualité ressurgie. Le sexe doit retrouver la chaleur de l'unité cosmique et divine, sans quoi il attise la blessure et la démesure de l'humain. L'être sans substance ni parole s'alourdit et se minéralise. L'unique révélation de la résurrection peut réussir à surmonter le déterminisme temporel, à faire resplendir le soleil de la nudité plus que le sombre sexe, dans le "nous" amoureux du couple. Car l'homme sans la femme reste aveugle et sourd, tout son champ est obscurci. Par contre, la fusion apporte la plénitude de la vue et de l'esprit hors de toute linéarité ou circularité. L'assimilation dans I'Un préserve de l'abolition du soi, de la néantisation et du divorce entre le sacré et le profane.

\section{Poésle (1972)}

A la suite de la réédition du texte revu et corrigé des quatre recueils de Fernand Ouellette, le livre Poésie rassemble plusieurs poèmes inédits et quelques autres déjà parus sous une première forme dans diverses revues. Événements (1968-71) comporte quatre sujets reliés chacun à un fait vécu, conférant un accent particulier à la préoccupation sociale du poète qui observe le quotidien. Dans "Bijou", le vertical et le circulaire, devenus des indices de mort, mènent vers l'abîme, le froid, le blanc et les oiseaux rapaces. Puis le corps de l'homme, fragmenté et douloureux, "remonte vers l'intégrité" (p. 224) dans “Aile 7B». (aile d'hópital, certes, mais aussi aile de l'opportunité salvatrice), grâce à la blancheur féminine qui apaise le feu et le noir. "Octobre 1970" prolonge cette souffrance dans l'historicité. II montre l'ébranlement du corps social et préfigure la critique des dirigeants politiques qu'approfondira en $1979 A$ découvert. La violence, appesantissant «l'homme jusqu'au silence» (p. 225), inflige une mortelle entaille à l'arbre du peuple. L'éblouissement ne sera reconquis que par "Le voyage" vers le bleu, l'audelà, la cime, l'esprit. Bénéficiant de la distance et de l'altitude, l'œil retrouve la cinématique du bris entre la convoitise de l'illumination et le danger de la dissémination.

Le Périple (1968) reprend entièrement cette problématique à l'intérieur d'un long poème oscillant depuis la volonté de se conquérir dans l'incessante douleur jusqu'à la réactualisation éphémère de l'harmonie béatifique. Le poète doit descendre vers l'humanité emmurée afin de l'alléger et de lui restituer la clairvoyance. Dans ce chaos d'en-bas, l'attrait ne dynamise guère l'âme massive du peuple malgré l'incandescence latente de l'idéal. Subsiste l'espoir interrogateur en l'aptitude de l'élan à dénouer l'immobile et le stérile. 
Car la substance aérienne corrode la matière, tend la parole vers l'avenir igné, restaure "l'acte de l'aile (...) dans sa permanence" (p. 234). Voici donc que la verticalisation reforme l'espace tout entier, transfigurant la lourdeur même dans le son et l'envol. Ainsi l'alliance tellurico-végétale se voit-elle sublimée par l'esprit, légitimée par la divinité en marche. Mais la vie est réduite au minimum en attente du jour, du souffle et du soleil. Seule la violence apportera la dilatation universelle, au risque de délaisser le cercle, le calme ilôt de l'œil. De cette unité potentielle surgit le lyrisme visionnaire d'une époque de sereine plénitude dont le destin mythique est mis en relief par les caractères italiques et la narration à l'imparfait. Dans ce lieu privilégié, où l'onirisme suscite l'hymne à la beauté et à la totalité primordiales, la psychée du couple magnétise la lumière. Toutefois, l'utopie se voit soudain interrompue, et le périple se poursuit inexorablement vers la destruction du pays.

Tout au long des textes qui forment La terre d'où... (1968-71), la parole de Fernand Ouellette atteint une densité exceptionnelle, toujours au bord du néant, du silence, du discontinu. Ecriture / fulgurance nécessitant une lecture / fulgurance, dans l'excès nucléique du langage. Raréfaction, concentration, suspension: ce livre catalyse la tension poétique au cœur même des mots qui portent la dialectique verbe-mutisme. A tout moment, le désir risque de faire éclater son expression dans l'au-delà, dans l'errance, dans la mort. Mais parce que le bleu natal fonde l'ère messianique, les mots tendent vers la réunification, restaurant en la femme la possible émergence cosmique. Le ciel demeure le lieu de la pureté, de la lucidité, juste à la limite du débordement passionnel. Près du gouffre froid et noir, fulgurante et spatiale, la conscience voisine l'incommunicabilité et le statisme. Quittant l'éblouissement, en effet, I'homme redevient sensible à l'odeur, à l'influence nocturne de l'organisme. II se referme, se coupe de sa dynamique pour se figer, pacifié, sur l'axe du monde qui favorise le retour du passé en abrogeant la fuite du temps. Si la branche offre une ouverture à l'inconditionné, un envol vers le bleu par l'embrasement, une nouvelle déchirure dans le sublime, aussitôt «l'arbre prévient la torture». II calme le sang, réduit la fracture en reconstituant la circularité du «dôme de verdoiement» (p. 246) hors de la violence de l'émotion.

La lumière menace l'intégrité du chercheur, le force à l'outrance, sature le psychisme et le regard, pour ne préserver que l'essence, la clameur, la sacralité de l'amour. La terre elle-même se fait lumineuse, et la flore présage la béance de l'infini. Pareille actualisation de la naissance provoque cependant l'incarnation dans la matière. Ainsi se perpétue la double fascination du terrestre et du céleste, de l'ange et de la chair, de l'envolée et de l'enracinement. Au centre même de la contraction s'esquisse la courbure, cette rencontre du vertical et de l'horizontal. Voici permis le périple initiatique de la tendresse, dans l'accalmie végétale de l'amour. Mais vite repris par la tourmente solaire, ce lieu frais de la fleur se trouve bientôt menacé par l'épaisseur. Et l'œil s'extrait d'un seul coup de la totalité incarnée, abandonnant la ronde corolle pour la tige dressée au-dessus de la terre. L'aile, le bleu, la verticalité, le ciel, l'inachèvement, forment autant de signes de la 
quête d'infini, quand la mort et la parole se confondent à l'ange et au mutisme. S'abolissent les contraires dans ces moments d'illumination, de foudroiement, qui dispersent tout abri. Mais l'homme se voit alors condamné à demeurer dans l'éternel clair-obscur, dans l'aphasie et le désert. La mémoire de la mer, ce lieu natal, peut seule favoriser la résurgence du pneuma de l'idéal, en provoquant une fois de plus la fusion des contraires, la sexuelle union de l'igné et de l'aqueux.

La pluie reprend encore l'oscillation depuis la ténèbre et le secret jusqu'à la disjonction de la continuité par la poussée, la rupture et l'irruption. Le souffle, la mouvance, la fraicheur, le feuillage, tous ces éléments mythiques d'une époque de paix irradiante, se voient enrayés par l'éclipse et le silence, par l'achèvement et la descente. Une telle évocation du néant entraîne à la pétrification, à l'opacité, au trépas. La parole et la mutité s'y jumellent. Malgré la polarité du principe spirituel, éolien et lumineux, s'impose l'autre póle, celui du trop plein passionnel, lourd et ombrageux. Dissolution de la totalité dans l'exigence sexuelle, dans la fragmentation du corps, mais aussi exil de l'âme, du souffle et de l'œil. Autant “la pensée se mutile» par “l'outrance dans l'infini / de lumière", autant "les mains mêmes se diluent contre la courbe / de la présence» (p. 255). La démesure est inhumaine, dans l'infini comme dans le fini, et pourtant elle reste le seul lieu de la poésie de F. Ouellette. Semblable excès ennoblit même la fabuleuse Sémélé, qui prétère l'érotique foudroiement du divin Soleil à la tendresse fraîche de l'ombre bien humaine. L'appel du désir triomphe de la tentation du paradis terrestre (ici encore décrite en caractères italiques). Malgré les avertissements de la sereine raison, l'amante se sacrifie au déchirement, à la folie du sublime, au féroce embrasement. Mais voilà que l'humain, s'imprégnant souverainement de cet éclat du Divin, ne s'en trouve pas dévoré. II transgresse au contraire la brûlure comme l'ombre et, oracle sur la montagne, subit la transfiguration de la fraîcheur dans la conjonction du chthonien et de l'ouranien, de l'audible et de l'inaudible, de la mort et de la naissance.

\section{Icl, ailleurs, la lumière (1977)}

En 1975, aux éditions Bourgignon + , paraissait le recueil Errances, composé de sept textes de F. Ouellette et de sept sérigraphies originales de Fernand Toupin. Ces sept poèmes sont redistribués dans la démarche beaucoup plus étoffée du livre de 1977, Ici, ailleurs, la lumière, dont la structure même se ressent de la tension entre l'immanence et la transcendance remarquée tout au long de cette étude. La première partie, L'ailleurs I, propose une géographie du corps ouvert et de l'åme en mouvement, une mutation du soleil et de la mer. La spiritualisation, toutefois, invoque aussitôt la mort. En effet, si l'oiseau allège le matériau jusqu'à l'émergence dans l'espace, le rêve et le bleu, une telle altitude se révèle insoutenable. L'immensité brise et désaccorde l'être qui doit réintégrer le vert terrestre pour se rassembler à nouveau. II découvre parmi le végétal les multiples signatures de l'univers mythique. Le paradis se réactualise dans la symbiose sol et soleil, 
où la fulgurance féconde sans détruire. Alors le vin permet de toucher au divin, la communion de l'air et de la terre restant assurée par la verticalisation de l'eau et du feu en l'accord transparent de l'arbre. Euphémisé dans la féminité, le lac protège l'homme de la brisure héroïque à laquelle invitent les cimes. Et le poète poursuit l'errance du feu à la cendre, de la sensorialité à la mysticité. Son regard est éveillé par les signes aériens qui divinisent l'humain, mais sa mémoire risque d'aspirer l'esprit dans le gouffre d'en-haut, dans "l'ailleurs".

La deuxième partie troque l'ailleurs géographique du voyage pour l'ailleurs spirituel de l'art, qu'il soit musical, littéraire, pictural ou sculptural. L'au-delà de la nature s'outrepasse lui-même dans un au-delà de la culture. Vecteur d'altitude, la tête "porte la flûte" (p. 23) et, par le souffle, appelle la feuillaison. Le son d'oiseau apparaît comme le multiplicateur de l'élévation originelle de l'arbre. La droiture végétale et musicale du tronc participe de la revitalisation de la pierre dans cette immatérialité, évoquant l'éros et la mort. Car le sexe féminin focalise d'une part le noir, la plaie, le piège et, d'autre part, l'infini, la chaleur, l'ouverture. La parole poétique possède ce pouvoir d'irradier, de sauver le besoin érotique en renversant "l'horizon vertical en la voix / qui le rachetait» (p. 24), en illuminant l'homme. Modulant l'air qui consume toute profanation, le verbe favorise l'incandescente extase de l'oiseau, l'emportement soudain de l'arbre et le redressement de la terre allégée. Quant à la couleur, elle fonde le lieu de l'origine par la médiation de la neige, de l'ange, de l'œil, de la fraîcheur et de la musique. Le mur lui-même "comme une aile s'éploie" dans le vert (p. 28) et monte vers la blancheur du silence, de l'errance, de la divinité. Bien sûr, la mort menace toujours le bleu, lorsque l'oiseau perce le cceur, ou que la nuit entoure le regard, ou que le piège obscur de la folie enserre l'âme et le ciel. Ce ciel qui s'arrête dans le silence, ce regard qui se brûle dans le jour, ce cœur tendu entre le départ et l'immobilité. Mais au-delà de l'oppression et du silence, perdurent les multiples élans de la spiritualité : le blanc, l'ange, l'allégement, la verticalité, la tension, la lumière, l'espace, Dieu, la pupille, la naissance, le regard, l'éclat, le jaillissement et le bleu.

Entraîné par une impulsion aussi irrépressible, Fernand Ouellette ne peut, à cause de la nature fondamentalement dialectique de son trajet poétique, que renoncer abruptement à «l'ailleurs" pour sombrer dans "l'ici ". La troisième partie du recueil, L'ici l, amorce grâce à la pierre et à la fleur (principes de rassemblement) ce retour à la matière. L'incendiaire frôle la mort sous la femme d'hiver, même si le poète, pressentant l'ombre, veut recommencer sa montée vers le bleu et le sommet. La Dame démembre le corps du mâle et enfouit son âme sous la braise et la pierre. Confinés à la racine et au silence, les amants sont privés de mobilité et de son. Ils croissent comme l'arbre vers l'abîme nocturne, car l'Éros disjoint le corps et son aura, ne projetant que l'ombre de la mort. Délaissé par la raison, l'homme n'a plus accès à l'éblouissement: l'aile s'alourdit, l'épine dorsale se còurbe, la lueur s'enferme. Alors l'extinction du phénix devient un indice de l'inertie intérieure. Le perpétuel envoûtement arrache l'arbre à la pierre, enfonçant le couple 
dans la ténèbre et l'ossification. Le soleil qui naguère favorisait l'ascension des sèves se trouve maintenant affecté par la mort verticale, la chute neigeuse de l'infini. Le blanc provoque la folie nyctomorphe, l'embourbement en le faux lumineux. II faut que la foudre frappe l'arbre pour restaurer le dépassement de la brûlure, principe d'unification de la mer, du feu et de la terre par l'intensité érotique. Seule cette concentration sur soi, noire fermeture au creux de l'épaisseur, assurera le triomphe de la conscience béatifique. Grâce à ce regard igné, l'amour rejoint l'oiseau pour procéder au départage de la terre et de la nuit. Ainsi la lumière parvient à agir comme facteur d'harmonisation du corps et du cosmos, annihilant le mal dans l'attrait ailé de la tendresse.

Comme la femme avait scellé l'homme dans la nuit, c'est elle également qui se voit chargée de libérer le sang de l'opacité, le bleu du gel, l'espoir de l'enceinte. Vecteur de la combustion, elle pourrait déstabiliser l'horizon et tirer momentanément l'homme du noir. Sans sa transparence, impuissant à trouver l'allégresse et l'unité, l'amant n'arrive pas à régénérer l'affection. Púpille fermée, désir fuyant, âme broyée, voyance emmurée, tous ces coefficients attestent de l'inaptitude du présent à actualiser le cheminement spirituel exigeant "que l'espace entier là-haut soit violé par la démesure" (p. 51). Condamné à s'étendre dans l'horizontalité fatale, le vivant ne trouve pas même une patrie où se réfugier. La mer, là barque, le soleil, refusent tour à tour leur asile. Le silence des ancêtres entraîne la perte de la parole généalogique pour les descendants lorsque le monde intime ne s'accorde pas à la lumière du peuple. Dans l'absence, le rêveur cherche désespérement l'embrasement vertical et les attributs ailés. Aux prises avec un spleen qui annule toute vigueur onirique, il se replie dans la nuit pour contrer l'errance. La neige et le blanc se dévoilent comme principes de séparation et de distanciation. Aussi paraitt-il nécessaire de renverser le pouvoir du gel afin de provoquer l'étincelle qui rendrait possible l'harmonie du «je” et de la clarté. "Mais le soleil bleu massivement s'étendait " (p. 56), empêchant l'allégement de l'esprit enfermé sous la glace. En effet l'ambiguïté solaire régit à la fois la dilatation de l'être vers l'espace de l'amour, et sa paralysie dans la fosse de la neige et du passé où se condense l'ombre. Le gel affecte le langage et la croissance du vivant, depuis la racine passionnelle jusqu'à l'incorporalité même. Mort-né, le poète a définitivement quitté la prodigalité primordiale.

Au cours de la dernière partie du livre, L'ici II, l'espoir soulève par instants ce lourd couvercle de l'hiver. Issus de l'empreinte énergétique, le feuillage, l'envol et l'oiseau apaisent l'arbre enraciné en lui apportant la mobilité et le surgissement. Ainsi le mot, malgré sa fermeture, sa résistance, son épaisseur, restaure imperceptiblement l'âme mythique dans l'instant hiératique. La parole poétique sourd du moment-limite de la condensation, du rassemblement dialectique depuis la distorsion de l'aile et la brūlure du bleu. La mort initiatique favorise donc le passage à la "profération nouvelle" (p. 63) dans la psychée préservant le feu natal de la parole, en deçà du corps qui propage la nuit et le froid. Mais cette ruine du quotidien affecte le cosmos entier: la mer se retire, le bleu s'embourbe, l'œil, le ciel et le soleil s'enfoncent 
sous terre. L'amour même se voit accablé par le sadisme, le haut désir par l'ombre, la pensée par la dispersion. L'oiseau, l'amant, l'air, la parole, la vision, le regard, témoignent tous de l'avortement de l'errance, si vitale au devenir intime du poète. L'arbre lui-même s'horizontalise dans la fin de l'élan. Et pourtant, la terre vibre à la dynamique ailée, conserve la tension éloignement-rassemblement, impose l'ailleurs dans l'ici. Le blanc retrouve son pouvoir éolien et permet aux défunts de se dresser et de parler d'une neuve voix. Persiste la déchirure verticale "de haut en bas" (p. 70), l'oscillation entre l'anéantissement par l'absolu prisme solaire et l'emprisonnement en la profusion végétale. Dans l'étau charnel, le regard quitte l'altitude et s'abaisse vers l'attrait marin, le sombre éros.

L'homme et l'arbre chassent alors l'oiseau, s'abandonnant à l'invasion du noir malgré le constant souhait de purification. Se succèdent les motifs de destruction, de paralysie, de lacération, tout meurt sous le déclin du soleil. L'oiseau lui-même répand la douleur de l'éthérisme, la suffocation dans l'espace, l'écrasement par la terre. Les morts abdiquent l'éclat, ne se lient plus au diurne. Ils s'enterrent dans l'immédiat et l'immobilisme de l'ici, sans se préoccuper de la mer, de l'aile, de l'air, figures mythiques de l'ailleurs. L'essentiel conflit entre cette immanence et cette transcendance ne s'incarnant plus qu'en l'arbre, l'esprit est évacué de la parole, de l'œil et du soi. Dans cette dissolution de toute pensée, dans cette métamorphose thériomorphe du ciel, dans cette extinction de la lumière, seul l'arbre demeure intouchable, comme en extase désirante. Grâce à l'axe immuable du tronc, l'esprit garde donc accès à la fulgurance par l'aile qui porte l'orant béatifié vers l'ardente inspiration. Toutefois, la voyance même se heurte à l'opacité qui enténèbre la pensée, le végétal, l'aile, la rose, l'œil enfin, ne laissant subsister de vie qu'en l'avenir. Acharné sous la mutité de la neige, le verbe igné du poème "se débat contre l'immobile" (p. 79), encore et toujours avide de sacralité. Mêmel'impuissance de la parole à se verticaliser quand le regard est brûlé par le visible ne doit qu'inciter l'homme à tendre davantage vers l'invisible. Mais "l'air à peine respirable" empêche la réalisation du salut dans l'ici, pour le confiner à «l'ailleurs, là-bas, en d'autres temps» (p. 81), puisque le cœur éteint empêche toute révolte, toute incarnation du projet en l'acte.

\section{A découvert (1979)}

Cette révolte se fait beaucoup plus tangible dans le dernier recueil de Fernand Ouellette. Le champ historique et politique qui motive la proferation impose une écriture plus directe, presque polémique, qui invite à relire les nombreux essais publî̀s par l'auteur. La structure poétique adopte un cheminement précis, répété presque tout au long du livre: énumération des difficultés d'un réel inacceptable, puis seule sortie possible dans l'espoir. L'aspect «engagé" de l'œuvre se voit mis en évidence par le nombre surprenant d'impératifs et surtout d'interrogatifs qui la parcourent. Le poète s'en prend aux multiples formes d'abus de l'autorité socio-politique, aux 
Généraux, aux Prédateurs, à l'Ordre et à l'Histoire. Il dénonce la torture, la répression, la dictature, la souffrance et la violence imposées à l'humanité par l'État sous tous les régimes. II en appelle à la conscience, puisque l'âme demeure l'indispensable lumière dans l'abîme, l'étincelle de la parole sur laquelle l'homme doit tout parier malgré la menace des puissants:

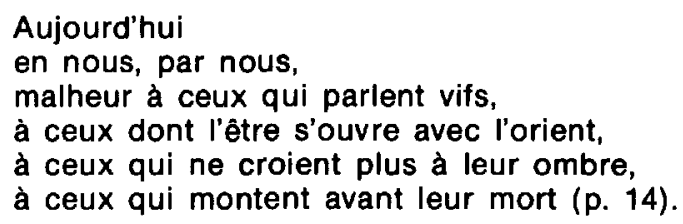

Pierre-Justin DÉRY

Université Laval 